\title{
Approval by Authorities Does Not Allow For Suboptimal Study Conduct but Offers New Academic Possibilities
}

\author{
Diana Lüftner \\ Department of Hematology, Oncology and Tumour Immunology, Charité University Hospital, Campus Benjamin \\ Franklin, Berlin, Germany
}

The year 2018 was the approval year of biosimilar versions of trastuzumab in Europe. While the economic advantage is clear and will be even more pronounced with presumably decreasing prices, the competition between pharmaceutical manufacturers is becoming more and more pronounced. Thus, the academic discussion 'pro and contra' biosimilar antibodies has shifted to 'Which is the best biosimilar trastuzumab?', with non-academic arguments being often used. Statements like 'We have the longest history of protein-based products.' or 'Our pathological complete remission rate is clinically relevantly higher than expected.' or 'We are the first to produce switch data.' are being brought forward.

In this context, it is worthwhile to rest a moment and have a closer look: Psychologically, it is understandable that oncologists like the idea of using a product that is being produced by a manufacturer with long-time experience in biomedical engineering and oncology per se. From a world-wide perspective, however, this is not relevant as standard methodologies can be transferred easily and with even improved, modernized quality controls. The rules for approval are identical for all providers, and as long as remission rates are within predefined margins and a biobetter can be formally excluded, we should not speculate about statistically not different response rates. While on the one hand, switching is not necessarily supported by oncological societies, in real-world practice with year-long treatment times, switches are a logical consequence. Switching data in clinical trials is necessary but can only be considered valuable if the observation times are long enough for adequate pharmacovigilance and survival evaluation.

\section{KARGER}

(ㅇ) 2019 S. Karger AG, Basel
In this unusual situation, clinical scientists should focus on scientific obligations and opportunities.

- Pharmacovigilance requires long-term observation and patient information!

- Survival counts highest and needs to be published!

- Gaps in the data set can now be closed!

In indications like chronic rheumatoid arthritis and ulcerative bowel disease, prospective and retrospective switching data have been gained. Thankfully, they did not show any relevant signal in terms of reduced efficacy or increased side effects or immunogenic events. Oncological patients can be treated safely but with adequate patient information only. When facing a life-threatening disease, transparency is helpful and warranted if we listen to the voice of patients' organizations. Every patient who is treated with a biosimilar antibody should know so and have had the chance to ask questions and, if desired, also to be kept on/initiated with the originator antibody.

The discussion in terms of influences on survival can be led in two directions. Like all glycosylated proteins, biosimilars could induce neutralizing antibodies, hereby reducing the therapeutic effect. Thus, long-term follow-up is necessary, especially in switching trials. Collecting shortterm survival rates, e.g. 1-year survival, is not the right way to address this question. On the other hand, and much more importantly, biosimilars with their refined antibodydependent cellular cytotoxicity (ADCC) controls could also have an improved outcome. So far, all agents have provided (more or less) the same response results in the predefined margins but still, slightly higher pathological complete remission rates in comparison to the originator product were seen. It is absolutely necessary that the patients in 
these trials are followed up for as long as possible to see an effect on survival, if there was one. And if so: This would not compromise the use of biosimilars but would be a testimonial for the progress in the manufacturing and control process of biological medicines.

Last but not least, we have the once-in-a-lifetime chance to fill scientific gaps that emerged in the development of monoclonal antibodies in oncology. For instance, we are missing data on the use of trastuzumab in metastatic HER2-positive gastric cancer: Apart from cisplatin and 5-fluorouracil/capecitabine, we have hardly any data on other combinations like irinotecan or taxanes. We are missing data on treatment beyond progression. Instead of repeating the TOGA trial in metastatic gastric cancer again and again by every biosimilar manufacturer, it would be excellent to support investigator-initiated trials in these situations. This will never lead to a formal approval (which will be given anyway by extrapolation from the breast cancer clinical trial results as a standard procedure) but to better clinical guidance of the patients.

Biosimilar antibodies will help to improve patient care individually and especially in societies with limited health care resources if we follow old rules in a new context.

\section{Disclosure Statement}

No conflict of interest. 